\title{
Video-Based Modeling of Dynamic Hair
}

\author{
Tatsuhisa Yamaguchi ${ }^{1, \star}$, Bennett Wilburn ${ }^{2}$, and Eyal Ofek ${ }^{3}$ \\ ${ }^{1}$ Kyoto University \\ yamaguti@vision.kuee.kyoto-u.ac.jp \\ ${ }^{2}$ Microsoft Research Asia \\ bennett.wilburn@microsoft.com \\ ${ }^{3}$ Microsoft Corporation \\ eyalofek@microsoft.com
}

\begin{abstract}
We present a method for creating realistic hair animation models based on videos of real hair in motion. We use an array of synchronized cameras to capture dynamic hairstyles. The hair structure and motion is modeled using an algorithm that grows each hair in segments from the root to the tip. Our algorithm generates hairs that are consistent with hair orientations observed in the input images and also enforces temporal smoothness constraints on the shape of the hair from frame to frame. Examples of synthesized hair models show the effectiveness of the method.
\end{abstract}

\section{Introduction}

As computer graphics technologies for rendering human skin and hair improve, accurately modeling the structure and motion of realistic hairstyles is becoming one limiting factor for creating lifelike renderings of people. Modeling hair is difficult because there are typically over 100,000 hairs on a human head, the hairs themselves are very thin, and the interactions can be complex. Moreover, there is currently no standard physically-based model for the mechanics of hair [1]. One approach to creating realistic dynamic hair motions, or studying the properties of hair to create a physically-based model, would be to capture the structure and motion of real, moving hair styles. The number of hairs and their fine structure make this quite challenging. The texture of hair is very self-similar, making it difficult to compute its three-dimensional structure or to track individual hairs from frame to frame.

We present a system for creating dynamic hair models by capturing the approximate structure and motion of real, moving hairstyles. Our system has two main components. The first is a set of cameras that record synchronized videos of moving hair. To prevent motion blur, we use strobe lights to capture images with a very short effective exposure time. The second component of the system is an algorithm that generates hair positions that are consistent with the input images. We strive to ensure that the hairs lie within the hair volume computed for each frame in the input videos, that their orientations are consistent with the two-dimensional orientations measured in the input views, and that their shapes do not change drastically from one time instant to the next. To our knowledge, our system is the first for automatically capturing dynamic hair styles.

\footnotetext{
^ This work was done while the author was visiting Microsoft Research Asia.
} 
In the next section, we discuss prior art for capturing static hair models. Section 3 describes our approach to capturing and modeling moving hair, and Section 4 details our specific implementation. In Section 5, we present experimental results. Finally, we close with a discussion of the limitations of our method and avenues for future work.

\section{Related Work}

For an excellent survey of hair modeling, simulation and rendering, we refer the reader to Ward et al. [1]. Several methods are capable of modeling static hairstyles from real images. Some use constraints provided by varying illumination [2]3], while others use multiple view geometry [45]. Wei et al. [5] capture many images of a static hairstyle from different viewpoints and measure 2D hair orientations in the images to determine 3D hair orientations. Their algorithm attempts to grow hairs, starting from the scalp, in such a way that the hair orientations match the input views. Paris et al. [6] recently demonstrated a system for capturing the shape and appearance of real hairstyles. They capture images from multiple viewpoints under multiple illumination conditions to create very high-quality hair shape and appearance models. They create geometric models using a plane sweep of light from video projectors and require images taken under different lighting conditions, so the method does not extend to moving hair.

\section{Image-Based Dynamic Hair Modeling}

We model hair using a hair-growing approach based on orientation information from multiple images, similar to Wei et al. [5]. To handle moving hair, though, we use a synchronized video camera array to simultaneously film the hair from many different viewpoints. We model each hair as a piecewise linear curve with equal length segments, and compute the 3D line segment positions for every frame in the input video. In this paper, "viewpoints" and "frames" refer to the spatial positions and the times at which we record the hair, respectively. Our cameras are synchronized such that at every frame, we simultaneously capture an image from every camera.

Applying Wei et al.'s algorithm independently to each frame in the multi-view video would not guarantee temporal consistency of the recovered hair geometry. The main contribution of this work is a hair growth algorithm that includes constraints for temporal continuity. Starting from the root for a given hair, the algorithm adds each new hair segment in parallel across all the frames of the video. Thus, starting from the root of a specific hair, we compute the endpoints of the first segment for that hair in all frames of the video, then the endpoints of the second segment for that hair in all frames of the video, and so on. For each segment, we optimize so that the hair growth direction at each frame is as consistent with the input images as possible, and so that the hair shape and motion varies smoothly from frame to frame.

In the next subsection, we describe the capture and preprocessing of multi-view videos of moving hair. The preprocessing includes generating visual hulls for the hair volume at each frame in the video and computing the two-dimensional hair orientation in the input images. Section 3.2 describes how we use this data to compute estimates of the 3D hair orientation, based on the input images, for any given point in the hair 
volume. In section 3.3 we explain how we use those estimates along with smoothness constraints for the hair shape and motion to create a temporally consistent model of the moving hairstyle.

\subsection{Capture and Preprocessing}

Multi-viewpoint video capture. We assume that $N_{\mathrm{f}}$ frames have been captured by $N_{\mathrm{c}}$ video cameras, meaning a total of $N_{\mathrm{f}} N_{\mathrm{c}}$ images are the input to the system. Hair often moves rapidly enough to cause motion blur in videos, which makes it much more difficult to estimate hair orientation and segment the hair from the background. We use a set of strobe lights synchronized to the cameras to create a very short effective exposure time. There are no other light sources in our system, and the strobe flash is intense but very brief, so the cameras capture blur-free, bright images regardless of their minimum exposure time.

Image segmentation. For every input image, we generate a pair of binary mask images that represent two different image segmentations. The first one is a silhouette mask which identifies the non-background region, including the subject's hair and skin. The second one is a hair mask which only segments the visible hair. We use the silhouette masks to create a visual hull [7] of the subject. The hair masks are used to limit the possible locations for hair roots and to restrict hair growth to regions where hair is present in the images.

Modeling the hair volume and scalp. Our hair growth algorithm requires a model of the hair volume for each frame in order to compute which cameras view a given hair tip and to know when the modeled hair grows out of the observed hair volume. We bound the hair volume by an inner scalp surface and an outer hair surface. We denote the hair surface in frame $k$ as $S_{\text {hair }}^{k}$ and the scalp surface as $S_{\text {scalp }}^{k} . S_{\text {hair }}^{k}$ is approximated using the visual hull reconstructed from the silhouette masks at each frame. The scalp surface, $S_{\text {scalp }}^{k}$, is approximated by fitting a generic head model to the video and the reconstructed visual hull. For a moving head, one must manually or automatically track the head motion. For simplicity, we do not attempt to track head pose in this work. Instead we capture moving hair on a still head, allowing us to focus purely on hair modeling.

Hair root generation. Our hair growth algorithm requires initial locations for the hair roots. We evenly distribute root positions over the scalp surface $S_{\text {scalp }}^{k}$ wherever the hair masks show hair. We assume that the root positions are fixed relative to the scalp model and generate them using the model from the first frame.

$2 D$ hair orientation. As we explain later, our hair growth algorithm estimates the observed three-dimensional growth direction at the tip of each growing hair. To do this, we must first compute the two-dimensional orientation of the local texture at each hair pixel, for every frame in all of the input videos. We adapted the filtering method developed by Paris et al[3] to compute hair orientation. Their method applies oriented edge detection filters at many angles, computes a filter response curve, and then selects the orientation at which the filter response is strongest. They produce a pair of values per pixel: a line orientation angle and a variance. The line orientation is an angle in $[0, \pi)$ that encodes the line direction in the image. The variance is a positive real value whose inverse represents the edge intensity and the certainty at each pixel. 


\subsection{Static Three Dimensional Hair Orientation}

As our hair modeling algorithm grows a single hair, it estimates the observed three dimensional hair orientation at the tips of the hair in every frame. This observed direction is computed independently for each frame, based solely on the input images from that frame, in a manner very similar to that of Wei et al. [5]. We briefly describe the method here, and refer the reader to their paper for more details.

We define a vector function for each frame $k$ that maps a point $P$ to its estimated orientation $D_{k}(P)$ :

$$
D_{k}(P)=o, D_{k}: \mathbf{R}^{3} \rightarrow\left\{o \mid o \in \mathbf{R}^{3},\|o\|=1\right\} \cup\{\phi\}
$$

Here, $\phi$ indicates that too few cameras viewed $p$, and thus we cannot estimate the hair orientation. The direction, $o$, is a three-dimensional vector of unit length. For each hair tip position and frame, we compute which cameras view that point, then triangulate the observed two-dimensional orientations to produce the estimated three-dimensional orientation.

Computing hair visibility. Given a hair tip at position $P$, we first compute which cameras view that point. For points on the hair surface, we can use the hair surface to determine visibility. The visibility at the surface can be computed once for each frame and stored. The visibility for points within the hair volume is defined to be the same as that of the closest point $P^{\prime}$ on $S_{\text {hair }}^{k}$. For such points, the projection point of $P$ on each image does not truly reflect the 3D orientation of the hair fiber at $P$, but we assume that the orientations at the surface are similar to the orientation within the volume. When we compute the 3D orientation of $P$, we average the orientation over the image area corresponding to the projection of a sphere that is centered at $P$ with radius $\overline{P P^{\prime}}$. This is a heuristic to obtain smoother orientations inside the hair volume.

Orientation triangulation. For each camera that views the hair tip, the computed 2D image orientation at the projection of $P$ defines a line in the image. The camera center and this line define a plane with unit normal $\boldsymbol{n}$ in 3D world coordinates. If the 3D hair orientation $\boldsymbol{o}$ matches the observed orientation in the image, the hair must grow along this plane, meaning that $(\boldsymbol{n} \cdot \boldsymbol{o})=0$. Each camera $j$ that views the hair tip yields a normal $\boldsymbol{n}_{j}$, and thus one linear homogeneous equation $\left(\boldsymbol{n}_{j} \cdot \boldsymbol{o}\right)=0$. If at least two camera views are available, and barring degenerate cases, we can solve for the $3 \mathrm{D}$ hair direction. If more are available, we compute an estimate of the true $3 \mathrm{D}$ hair direction based on the observed orientations and accounting for the confidence in the observations. We solve for the orientation $o$ by minimizing

$$
\Sigma_{j} \frac{1}{\sigma_{j}^{2}}\left(\boldsymbol{n}_{j} \cdot \boldsymbol{o}\right)^{2}, \text { subject to }\|\boldsymbol{o}\|=1,
$$

where $\sigma_{j}$ is the variance of the orientation filter response curve at that position. Eq 2 is a linear optimization problem that is efficiently solved by singular value decomposition. The direction for $o$ is ambiguous; if $o$ is a solution, $-o$ is, too. This ambiguity is resolved 
by choosing the direction that forms the largest angle with the computed hair growth direction of the previous segment.

\subsection{Temporally Consistent Hair Models}

Once we have the hair volume model, 2D orientation information for the input images, and the initial hair root locations and growth directions, we can begin to grow hair segments from the roots. We add segments one by one to each hair and determine the growth direction of each new segment in all frames by optimizing an energy function that has terms for data consistency and smoothness. Figure 1 shows an example of the known and unknown quantities during the optimization for segment $l$ on a hair. The position of the current hair tip is known for all frames (shown as $P_{k}^{l-1}, P_{k+1}^{l-1}, P_{k+2}^{l-1}, \ldots$ ) as well as the previous growth directions $d_{k}^{l-1}$ for all $k$. These quantities are expressed in world coordinates. As described in the previous subsection, we can compute an estimate of the observed 3D hair orientation for each frame, shown as $\boldsymbol{o}_{k}^{l}, \boldsymbol{o}_{k+1}^{l}, \boldsymbol{o}_{k+2}^{l}, \ldots$. To add a new hair segment to the hair, we simultaneously solve for the growth direction of the segment in all frames, i.e. $\boldsymbol{d}_{k}^{l}$ for all $k$. Because the segment length is fixed, this also determines the new hair tip positions, $P_{k}^{l}$ for all $k$. Not shown in the figure are $P_{k}^{0}$ and $\boldsymbol{d}_{k}^{0}$, the root position and the initial growth direction for the hair, and $L_{\mathrm{s}}$, the length of each hair segment (a user-specified parameter for the algorithm).

Algorithm 1 summarizes our method in pseudocode. Each hair is grown independently from the others. We attempt to ensure that each new hair segment's 3D orientations in each frame are consistent with the observed 2D orientations in the videos, that the hair stays within the observed hair volume, and that the hair shape varies smoothly from frame to frame. We formulate these constraints as minimizing an energy with terms for orientation consistency, shape smoothness, motion smoothness, curvature and surface potential (described below) as follows:

$$
E\left(\left\{\boldsymbol{d}_{k}^{l}\right\}_{k=0,1, \ldots, N_{\mathrm{f}}-1}\right)=E_{\mathrm{o}}+\alpha_{1} E_{\mathrm{s}}+\alpha_{2} E_{\mathrm{SS}}+\alpha_{3} E_{\mathrm{C}}+\alpha_{4} E_{\mathrm{B}}
$$

Here, $\alpha_{1}, \alpha_{2}, \alpha_{3}, \alpha_{4} \in \mathbf{R}$ are manually specified weights for each term.

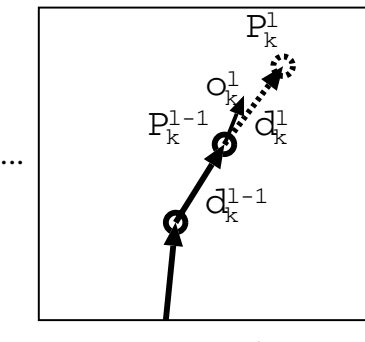

Frame k

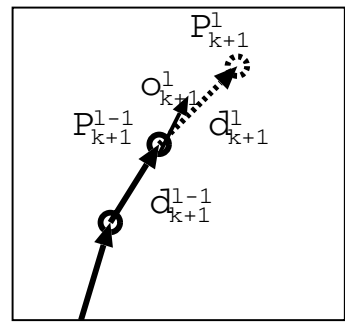

Frame $\mathrm{k}+1$

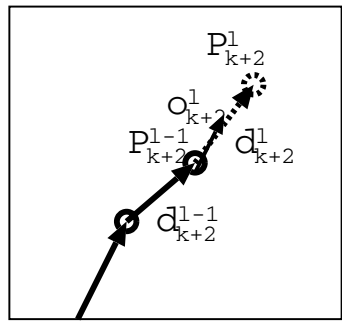

Frame $\mathrm{k}+2$

......... Optimizing at this step

$\longrightarrow$ Already generated

Fig. 1. Notation for the hair growing algorithm. See the text for details. 


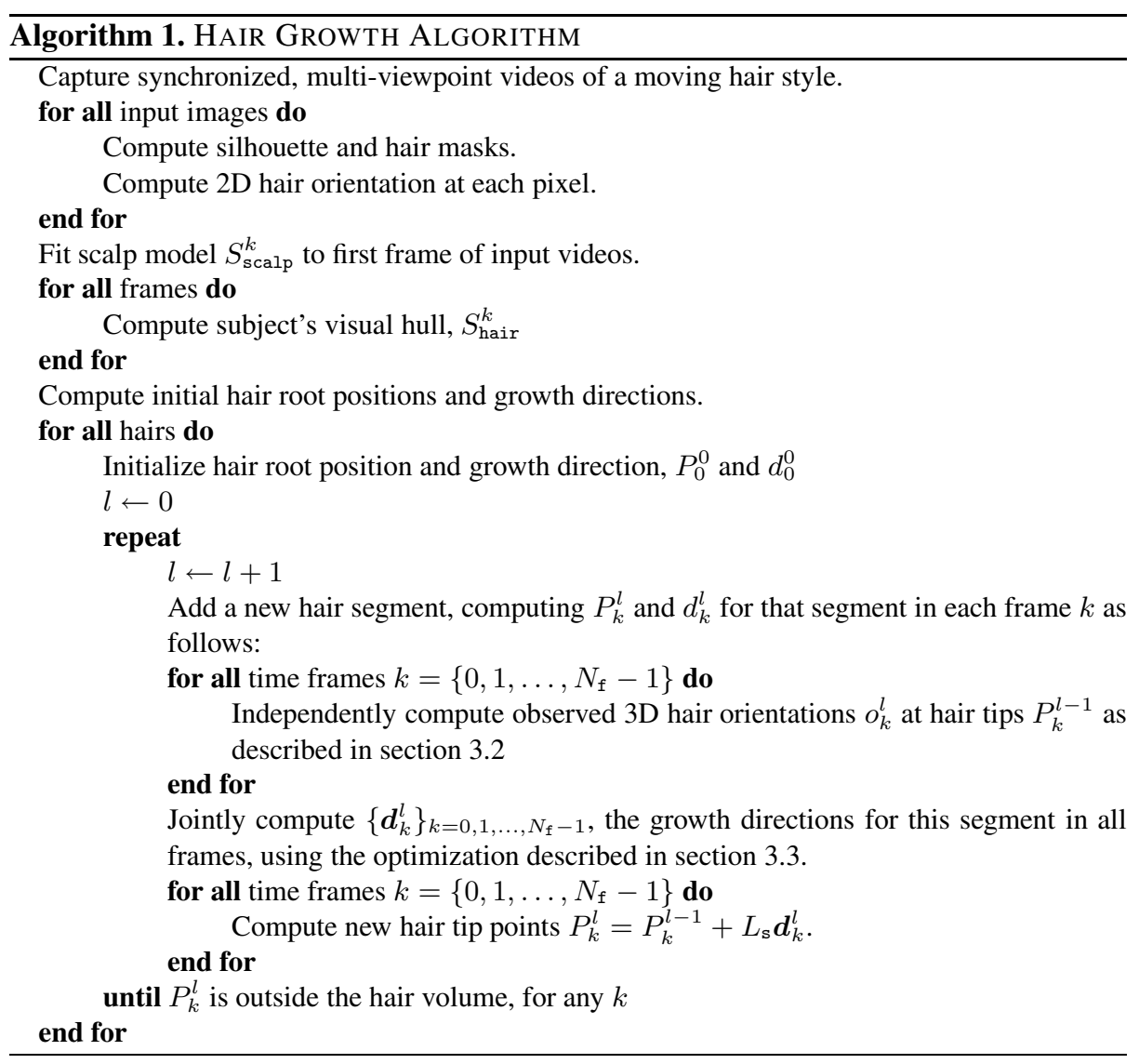

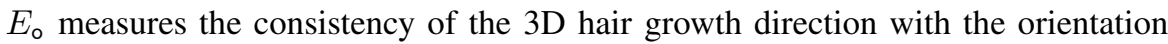
estimated from the images:

$$
E_{\circ}=\sum_{k=0}^{N_{\mathrm{f}}-1} \operatorname{dist}\left(\boldsymbol{d}_{\boldsymbol{k}}^{\boldsymbol{l}}, \boldsymbol{o}_{\boldsymbol{k}}^{\boldsymbol{l}}\right),
$$

Here, $\operatorname{dist}(a, b)=(1-a \cdot b)^{2}$ is a distance function for a pair of unit vectors which is always non-negative. The value of this function is defined as 0 when either $a$ or $b$ is $\phi$, i.e. when the orientations $\boldsymbol{o}_{k}^{l}$ are not available. This occurs, for example, when too few cameras view the hair tip. In this case, we set Eq4 to a constant value (for some frames $k$ ), and the growth directions are optimized based only on the remaining terms.

$E_{\mathrm{s}}$ is a motion smoothness term that represents the difference in growth direction from frame to frame:

$$
E_{\mathrm{s}}=W(l) \sum_{k=1}^{N_{\mathrm{f}}-1} \operatorname{dist}\left(\boldsymbol{d}_{\boldsymbol{k}}^{l}, \boldsymbol{d}_{k-1}^{l}\right)
$$


where $W(l)$ is positive and non-increasing function of $l$. This ensures that growth directions are similar near the root, but can vary more and more towards the end of the hair.

$E_{\mathrm{SS}}$ ensures that the hair shape is similar from frame to frame:

$$
E_{\mathrm{SS}}=\sum_{k=1}^{N_{\mathrm{f}}-1} \operatorname{dist}\left(\boldsymbol{d}_{\boldsymbol{k}}^{\boldsymbol{l}}-\boldsymbol{d}_{\boldsymbol{k}}^{\boldsymbol{l - 1}}, \boldsymbol{d}_{\boldsymbol{k}-\mathbf{1}}^{\boldsymbol{l}}-\boldsymbol{d}_{\boldsymbol{k}-\mathbf{1}}^{\boldsymbol{l - 1}}\right)
$$

To ensure that hairs do not double back on themselves and that the shape is realistic, we discourage highly curved hair with a curvature cost:

$$
E_{\mathrm{C}}=\sum_{k=0}^{N_{\mathrm{f}}-1}\left(\frac{R_{0} \arg \left(\boldsymbol{d}_{\boldsymbol{k}}^{\boldsymbol{l}}, \boldsymbol{d}_{\boldsymbol{k}}^{\boldsymbol{l - 1}}\right)}{L_{\mathrm{s}}}\right)^{4}
$$

$R_{0}$ is the threshold curvature expressed as a radius, typically $20 \mathrm{~mm}$, and $\arg \left(\boldsymbol{d}_{\boldsymbol{k}}^{l}, \boldsymbol{d}_{\boldsymbol{k}}^{l-1}\right)$ is the angle between the two vectors, in radians. The curvature cost approximates the fourth power of the sine of the angular difference in hair directions from segment to segment. Thus, it is small in areas of low curvature but grows rapidly as the curvature increases.

The final term, $E_{\mathrm{B}}$, is a surface potential which prevents hairs from leaving the hair volume:

$$
E_{\mathrm{B}}=E_{\mathrm{B}}^{\mathrm{S}}\left(l_{d}\left(P_{k}^{l-1}+L_{\mathrm{s}} \boldsymbol{d}_{k}^{l}\right)\right)+E_{\mathrm{B}}^{\mathrm{H}}\left(l_{h}\left(P_{k}^{l-1}+L_{\mathrm{s}} \boldsymbol{d}_{k}^{l}\right)\right)
$$

In this equation, $l_{\mathrm{d}}(P)$ is the "depth" inside the hair surface, meaning the distance from $P$ to the nearest point on $S_{\text {hair }}^{k}$. It is negative if the point is outside the hair surface. $l_{\mathrm{h}}(P)$ is the "height" from scalp surface, i.e. the distance from $P$ to $S_{\mathrm{scalp}}^{k}$. It is negative if the point is inside the scalp surface. The surface potential functions, $E_{\mathrm{B}}^{\mathrm{S}}$ and $E_{\mathrm{B}}^{\mathrm{H}}$ are ramp functions. This surface potential curve is shown graphically in Figure 2 .
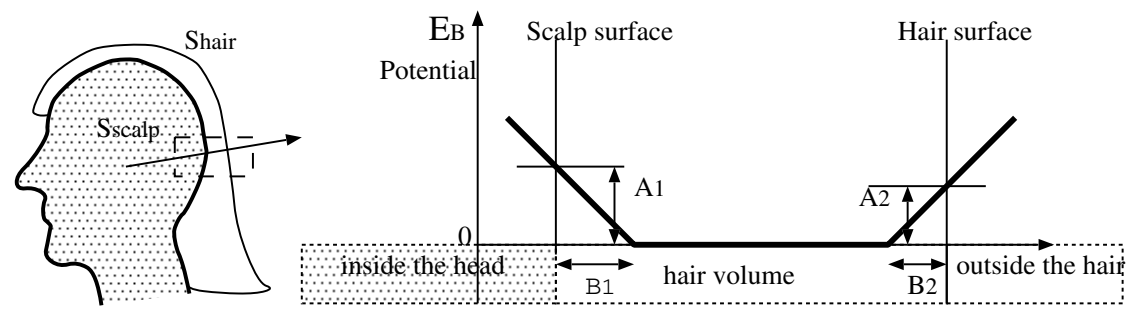

Fig. 2. The surface potential function that encourages hairs to grow only within the hair volume. The potential is a function of the distances from a point $\mathrm{P}$ to the nearest points on the hair and scalp surfaces, $S_{\text {hair }}^{k}$ and $S_{\text {scalp }}^{k} . A_{1}, A_{2}, B_{1}$, and $B_{2}$ specify the potential ramp functions. 


\section{Implementation}

Multiple-viewpoint video capture. We use twelve synchronized PointGrey Research Dragonfly cameras to capture our videos. The cameras are on a hexagonal frame with a two meter diameter, shown in Figure 3. The subject is set in the center of the frame. Due to the limited number of cameras, we can only observe one side of the subject with enough cameras to accurately estimate hair orientations. Thus, for our experiments we reconstruct only one side of the hair volume. The videos are VGA $(640 \times 480$ pixel) resolution at 30 frames per second. For illumination, we use six SHIMPO DT-311A stroboscopes synchronized to the cameras. The walls and floor of the camera frame are covered with green fabric so we can use green screen matting to segment the subject in the images. The cameras are geometrically calibrated using Zhang's method[8].

$2 D$ orientation map computation. To compute the orientation at each pixel in each image, we apply two Canny-like first derivative of Gaussian filters with different wavelengths $(\lambda=2$ and $\lambda=4)$ and apply them at discrete angles for each pixel. The angle at which the filter gives the highest response is the orientation for that pixel. We use the inverse of the variance of the filter response versus angle as a confidence measure for the orientation.

Image segmentation. We use very simple chroma keying in the HSV color space for foreground/background and skin/hair segmentation. We manually select the foreground hue mean and threshold values. All pixels whose hue is within the threshold of the mean are labeled foreground. Very dark pixels are also assumed to be foreground. We create the visual hulls at each frame using the method of Franco and Boyer [9]. To create scalp models, we manually fit a generic model to the first frame of the input data. For experiments using a mannequin head and wig, we use images without the wig to make a visual hull for the scalp model. When we do not use a wig, we manually fit a generic scalp model to the subject.
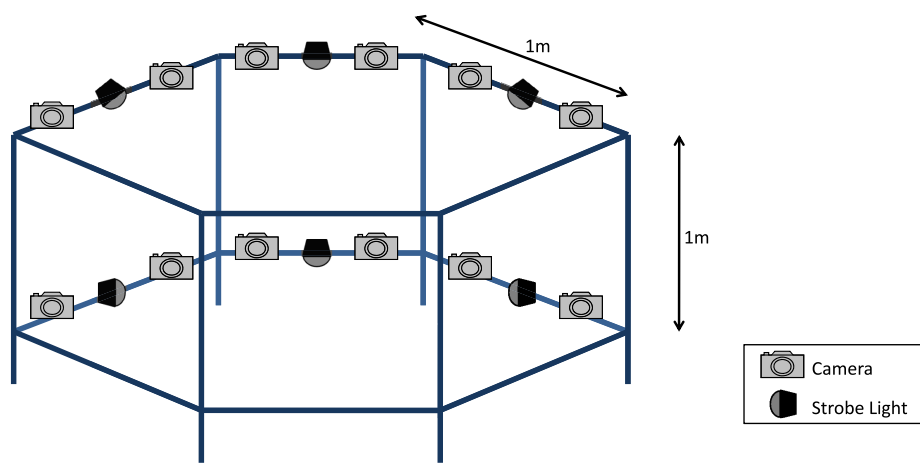

Fig. 3. Our camera array setup. Twelve cameras on one side of a hexagonal frame film the subject. Ideally, we would use more cameras to fully surround the subject. Strobe lights synchronized to the cameras provide intense, brief flashes of light, leading to images with negligible motion blur. 
Hair root formation. We generate hair roots on the scalp surface as follows. First, we use Saff and Kuijlaar's algorithm [10] to generate evenly distributed samples on a unit sphere. These unit vectors can be considered sampled directions from the origin. We place this origin roughly in the center of $S_{\mathrm{scalp}}^{k}$ for the first frame of the video, cast rays from the origin in each sampled direction, and compute where they intersect $S_{\mathrm{scalp}}^{k}$. We check the projection of each intersection point into the hair mask from the most frontoparallel camera view. If the projection lies on a pixel for which the mask indicates hair, we use the intersection point as a hair root position.

We must also set an initial hair growth direction, $d_{0}^{0}$, for each root. We use a heuristic to set this properly so hairs grow down the head, not up. As just described, each root corresponds to a sample point (or direction) $P$ on the unit sphere. Let $P=(x, y, z)$, with the positive $z$ axis pointing up vertically. For hair roots corresponding to $z<0$, $d_{0}^{0}$ is set to $(0,0,-1)$. Otherwise, $d_{0}^{0}$ is the unit vector in the plane orthogonal to $P$ with the most negative $\mathrm{z}$ component.

Hair shape reconstruction. We found that choosing $L_{\mathrm{s}}=0.8 \mathrm{~mm}$ generates visually smooth hairs with reasonable computation time. For each new hair segment, we solve the minimization problem in Section 3.3 using the Levenberg-Marquardt algorithm [11/12]. The initial value for each segment is set as follows:

$$
{ }^{\text {init }} \boldsymbol{d}_{k}^{l}= \begin{cases}\boldsymbol{o}_{k}^{l} & \left(\text { if } o_{k}^{l} \neq \phi\right) \\ { }_{\text {init }}^{l} \boldsymbol{d}_{k-1}^{l} & \text { (if } o_{k}^{l}=\phi \text { and k>0) } \\ \boldsymbol{d}_{k}^{l-1} & (\text { otherwise }) \\ & \left(k=0,1, \ldots, N_{\mathrm{f}}-1\right)\end{cases}
$$

Noisy hair removal. Our algorithm occasionally produces hairs whose shape and motion are noisy. We remove them based on the following noise measure:

$$
E_{\mathrm{N}}=\Sigma_{l=0}^{L-1} \frac{1}{L_{\mathbf{s}}(l+1)} \Sigma_{k=0}^{N_{\mathrm{f}}-3}\left\|-P_{k}^{l}+2 P_{k+1}^{l}-P_{k+2}^{l}\right\|,
$$

where $L$ is the number of segments in the hair. $E_{\mathrm{N}}$ is the sum of the second derivatives of the hair segment endpoints. Intuitively, it measures the total energy required to realize the hair motion. This measure tends to be large for hairs that vibrate vigorously due to noise. Hairs with noise measures above a threshold value are eliminated. Once the hair model has been generated, the noise energy threshold can be adjusted interactively to produce visually pleasing results.

\section{Results}

We captured videos of an artificial hairstyle using a wig on a mannequin head, with a fan to make the hair move. In this way, we could concentrate solely on hair modeling. Because of our limited number of viewpoints, we reconstruct only the right side of the hairstyle. Figure 4 shows example input images and the resulting hair motion model for videos of a wig with short, multi-colored hair. The execution time for this result was nearly a day. This is more than a factor of $N_{f}$ increase over the static hair execution 

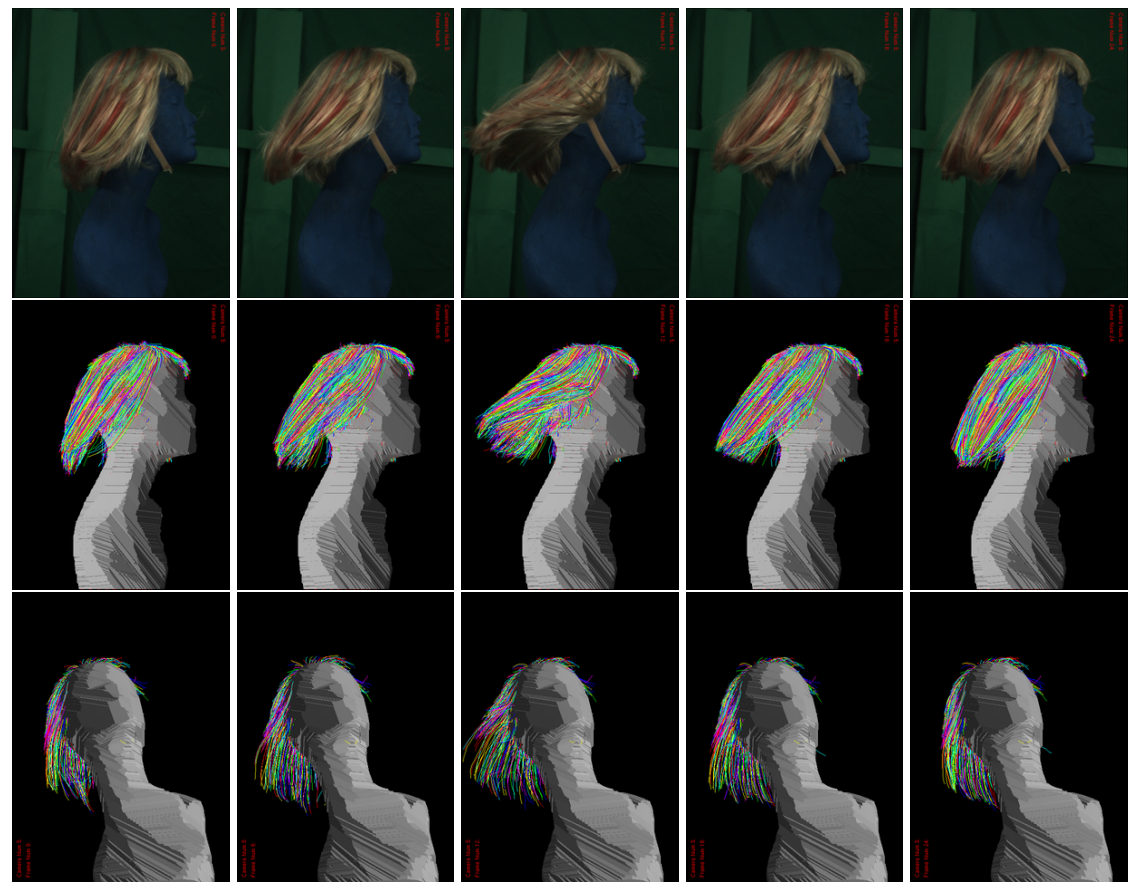

Fig. 4. Modeling result for artificial hair on a mannequin head. These images show every sixth frames from a thirty frame sequence. Top row: real images from one camera view. Middle row: reconstructed hair rendered from the same view point and camera parameters show motion similar to the original images. The hair fibers are colored with the same value in all images for easier identification. Bottom row: Rendering the hair from a new viewpoint shows that we have recovered the three dimensional structure and motion of the hair. Some fibers were not drawn to make the visualization clearer.

time because of the Levenberg-Marquardt optimization for each hair segment. About 5000 hair fibers were generated for the 30-frame sequence. The results show that the recovered hair shape and motion is similar to the actual hair behavior.

One limitation we found on datasets with more complicated hairstyles and motions is that clumps of long hairs spread out in some frames, increasing the probability that the modeled hair strands will grow out of the hair volume. When this happens, the hair growth is terminated prematurely, and the modeled hairs are shorter than the real ones. This is a limitation of our method; as the hair shape or motion gets more complex, the chance of modeled hairs growing out of the hair volume increases.

\section{Conclusions and Future Work}

In the near future, we would like to address some of the resource limitations of our system. Adding more cameras would enable us to observe and model all of the hair on 
the subjects, and would also increase the robustness of our orientation data. We would also like to incorporate head tracking to capture real human subjects.

One fundamental limitation of our approach is that it cannot recover from early errors estimating hair growth directions. Wei et al.'s method also suffers from this drawback, but for static hairstyles, the resulting hair model is often plausible. For a dynamic hairstyle, however, early errors in the hair growth directions for different frames increase the chance that the hair will not resemble any of the actual filmed hairs. Such incorrectly modeled hairs leave the hair volume prematurely in some frames or simply do not match the input data. Ideally, we would iteratively refine the entire length of each modeled hair instead of simply growing the hair from the tip, but this would dramatically increase the time required to create the model.

We have presented the first system for automatically creating models of moving hair from multiple viewpoint video of real, dynamic hairstyles. Our method has produced compelling results for simple hairstyles and motions, and we hope to see others build on this work. Creating more accurate models, and modeling more complicated hairstyles and motions, would have many benefits beyond simply capturing and reproducing existing hair motions. We anticipate capturing hair motions in order to infer physical models for the dynamic behavior of real hairstyles. This would not only deepen our understanding of how hair behaves, but also lead to easily controllable, realistic, dynamic hair models.

\section{Acknowledgements}

The authors would like to thank Sing Bing Kang and YiChen Wei for helpful discussions, and YeBin Liu for assistance with our capture system.

\section{References}

1. Ward, K., Bertails, F., Kim, T., Marschner, S., Cani, M., Lin, M.: A survey on hair modeling: Styling, simulation, and rendering. IEEE Transactions on Visualization and Computer Graphics 13(2), 213-234 (2007)

2. Grabli, S., Sillion, F., Marschner, S., Lengyel, J.: Image-based hair capture by inverse lighting. In: Proc. Graphics Interface, pp. 51-58 (May 2002)

3. Paris, S., Briceno, H.M., Sillion, F.X.: Capture of hair geometry from multiple images. ACM Transactions on Graphics 23(3), 712-719 (2004)

4. Kong, W., Takahashi, H., Nakajima, M.: Generation of $3 \mathrm{~d}$ hair model from multiple pictures. In: Proc. Multimedia Modeling, pp. 183-196 (1997)

5. Wei, Y., Ofek, E., Quan, L., Shum, H.: Modeling hair from multiple views. ACM Transactions on Graphics 24(3), 816-820 (2005)

6. Paris, S., Chang, W., Jarosz, W., Kozhushnyan, O., Matusik, W., Zwicker, M., Durand, F.: Hair photobooth: Geometric and photometric acquisition of real hairstyles. ACM Trans. on Graphics 27(3) (2008)

7. Laurentini, A.: The visual hull concept for silhouette based image understanding. IEEE Trans. on Pattern Analysis and Machine Intelligence 2(16), 150-162 (1994)

8. Zhang, Z.: A flexible new technique for camera calibration. IEEE Transactions on Pattern Analysis and Machine Intelligence 22(11), 1330-1334 (2000) 
9. Franco, J., Boyer, E.: Exact polyhedral visual hulls. In: Proceedings of the Fourteenth British Machine Vision Conference, Norwich, UK, pp. 329-338 (September 2003)

10. Saff, E.B., Kuijlaars, A.: Distributing many points on a sphere. Mathematical Intelligencer 19(1), 5-11 (1997)

11. Levenberg, K.: A method for the solution of certain non-linear problems in least squares. The Quarterly of Applied Mathematics 2, 164-168 (1944)

12. Marquardt, D.: An algorithm for least-squares estimation of nonlinear parameters. SIAM Journal on Applied Mathematics 11, 431-441 (1963) 